University of Wollongong

Research Online

Faculty of Health and Behavioural Sciences -

Papers (Archive)

Faculty of Science, Medicine and Health

$1-1-2008$

\title{
Reflex changes associated with anticipatory postural adjustments preceding voluntary arm movements in standing humans
}

\author{
Siddharth Vedula \\ Neuromuscular Control Lab, McGill Uni, Montreal \\ Paul J. Stapley \\ University of Wollongong, pstapley@uow.edu.au \\ Robert E. Kearney \\ Neuromuscular Control Lab, McGill Uni, Montreal
}

Follow this and additional works at: https://ro.uow.edu.au/hbspapers

Part of the Arts and Humanities Commons, Life Sciences Commons, Medicine and Health Sciences Commons, and the Social and Behavioral Sciences Commons

\section{Recommended Citation}

Vedula, Siddharth; Stapley, Paul J.; and Kearney, Robert E.: Reflex changes associated with anticipatory postural adjustments preceding voluntary arm movements in standing humans 2008, 4523-4526.

https://ro.uow.edu.au/hbspapers/861

Research Online is the open access institutional repository for the University of Wollongong. For further information contact the UOW Library: research-pubs@uow.edu.au 


\title{
Reflex changes associated with anticipatory postural adjustments preceding voluntary arm movements in standing humans
}

\author{
Abstract \\ Dynamic changes in human stability, such as those induced by upper body movements, are preceded by \\ anticipatory postural adjustments (APAs) in the rest of the body. We measured the excitability of the \\ stretch reflex of the triceps-surae muscle group during APAs associated with unilateral right arm raises in \\ standing humans. Our results demonstrate that reflex excitability and underlying muscle activity are \\ linked during the APA period, but that they differ in their relative timing. This supports the idea that \\ reflexes are controlled independently of muscle activation.

\section{Keywords} \\ arm, movements, reflex, standing, anticipatory, humans, associated, postural, changes, adjustments, \\ preceding, voluntary

\section{Disciplines} \\ Arts and Humanities | Life Sciences | Medicine and Health Sciences | Social and Behavioral Sciences

\section{Publication Details} \\ Vedula, S., Stapley, P. J. \& Kearney, R. E. 2008, 'Reflex changes associated with anticipatory postural \\ adjustments preceding voluntary arm movements in standing humans', in 30th Annual International IEEE \\ EMBS Conference, 20-24 August, Vancouver, British Columbia, Canada, IEEE Engineering in Medicine and \\ Biology Society. Conference Proceedings, vol. 2008, pp. 4523-4526.
}




\title{
Reflex Changes Associated with Anticipatory Postural Adjustments Preceding Voluntary Arm Movements in Standing Humans
}

\author{
Siddharth Vedula, Paul J. Stapley, and Robert E. Kearney, Fellow, IEEE
}

\begin{abstract}
Dynamic changes in human stability, such as those induced by upper body movements, are preceded by anticipatory postural adjustments (APAs) in the rest of the body. We measured the excitability of the stretch reflex of the triceps-surae muscle group during APAs associated with unilateral right arm raises in standing humans. Our results demonstrate that reflex excitability and underlying muscle activity are linked during the APA period, but that they differ in their relative timing. This supports the idea that reflexes are controlled independently of muscle activation.
\end{abstract}

\section{INTRODUCTION}

$\mathrm{H}$ UMAN stance may be modeled biomechanically as an inverted pendulum pivoting about the ankle joint [1]. The body has a high center of mass (COM) and a small support base at the feet, so the pendulum is fundamentally unstable as gravity acting on the COM will cause it to topple over. Studies of quiet stance have shown that the center of vertical pressure (COP) at the feet is constantly displaced so that the vertical projection of the COM is maintained slightly anterior to the ankle joint [1]. In summary, relaxed human posture is unstable, with constant sway.

Electromyographic (EMG) recordings from the muscles of the triceps-surae (TS) complex show that they actively contract in quiet stance [2] producing the torques needed to counteract gravity. Along, with the flexor muscle of the ankle, they have been identified as the prime effectors regulating sway. It is thought that postural sway is controlled by a combination of the passive mechanical visco-elastic properties of the ankle muscles, tendons and ligaments [1] and active control from the central nervous system (CNS) incorporating vestibular, visual and proprioceptive information [3]. There has been much debate about the

Manuscript received April 7, 2008. This work was supported in part by grants from the Canadian Institutes of Health Research (CIHR) and the Natural Sciences and the Natural Sciences and Engineering Research Council of Canada (NSERC).

V. Siddharth is with the Department of Biomedical Engineering, McGill University, 3775 Rue University, Montreal, QC, H3A 2B4 CANADA (Phone: 514-398-4400 ext.00425; fax: 514-398-7461; e-mail: siddharth.vedula@mail.mcgill.ca).

P. J. Stapley is with the Department of Kinesiology and Physica] Education, McGill University, 475 Avenue Des Pins Ouest, Montreal, QC, H2W 1S4 CANADA (e-mail: paul.stapley@mcgill.ca).

R.E. Keamey is with the Department of Biomedical Engineering, McGill University, 3775 Rue University, Montreal, QC, H3A 2B4

CANADA (email: robert.keamey@mcgill.ca). underlying neural mechanisms. It has been postulated that active CNS control involves both feedback (reactive) $[3,4]$ and feedforward (predictive) mechanisms [5].

The feedback mechanism of primary postural significance is the stretch reflex. Stretching a muscle will initiate a reflex arc that that will cause it to contract and return to its resting length. It is thought that the TS stretch reflex plays an important role in the control of sway by counteracting forward lean; studies have shown that it can contribute significant stiffness (i.e. resistance to sway) to the ankle [6] and that it may be centrally modulated [7] independently of passive contributions.

On the other hand, the core idea behind feedforward mechanisms is that the CNS uses anticipatory control to maintain stability and employs strategies optimized on the basis of past experience. There is debate about the extent to which such strategies are used during quiet stance. However, in situations which involve internally generated perturbations, it is widely accepted that feedforward postural control is used. Thus, it has been shown that for a range of voluntary arm, trunk or leg movements, that there are anticipatory postural adjustments (APAs) in other body segments, where specific motor programs of postural muscles precede those of the focal muscles required for the movement [8].

To date, no one has examined how the stretch reflex is modified during these APAs. A common task used in postural control studies is the unilateral arm raise, during which the TS shows a characteristic APA. Therefore, the aim of our study was to quantify stretch reflex changes in the TS during the APA associated with a voluntary unilateral arm raise, and to compare the relative time course of changes in reflex excitability and associated muscle activity.

\section{METHODS}

\section{A. Experimental Apparatus}

Fig. 1 shows a typical subject on the experimental apparatus made up of four main components.

A) A custom-built, bilateral, electro-hydraulic actuator comprising two rotary foot pedals equipped with load cells, potentiometers and torque sensors. This was used to apply short position perturbations to the right ankle and measure the responses. 
B) A custom-built, adjustable aluminum frame housing a visual cue (light emitting diode- LED), an audio cue (piezoelectric buzzer), and a movement target (Double Pole Double Throw Switch).

C) An 8 channel differential EMG (Gain 1000, Bandwidth 20-2000 Hz) system (Delsys Bagnoli) was used to record muscle activity from the main postural muscles of both legs (Soleus, Lateral and Medial Gastrocnemius, and Tibialis Anterior) and the primary focal muscle of the arm involved in the task (Right Anterior Deltoidus). An $\mathrm{Ag} / \mathrm{AgCl}$ reference electrode ( $3 M \operatorname{Red} D o t$ ) was placed on the right knee cap.

D) A single-axis inclinometer (Microstrain FAS-G) fixed to a splint (Formedica Ergo-forme) on the right wrist and oriented orthogonally to the radial bone was used to measure arm kinematics. A custom-made cast prevented elbow flexion.

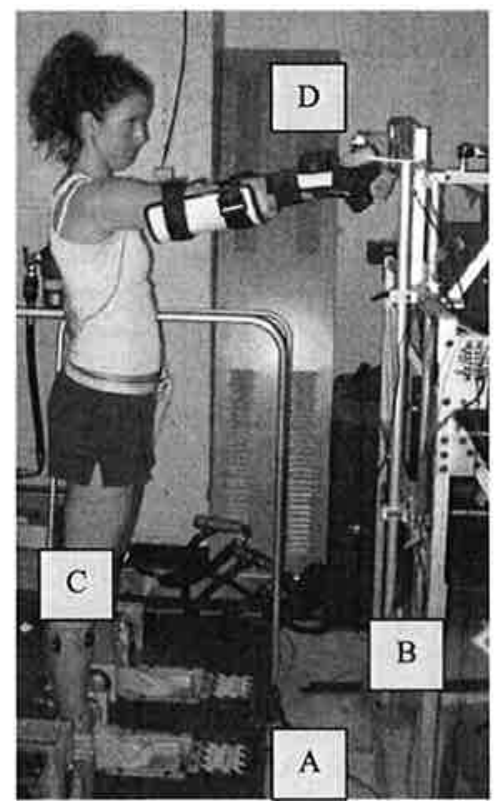

Fig. 1. A typical subject executing a voluntary arm movement while standing on the actuator. A) Bilateral electro-hydraulic actuator fitted with two foot pedals and torque, position and load transducers. B) Aluminum target frame holding visual and audio cues, torque feedback meter and target switch. C) Surface EMG electrodes on postural and focal muscles. D) Inclinometer used to measure arm position.

\section{B. Experimental Protocol}

\section{I) Defining the operating point}

Subjects were instructed to stand on the two foot pedals and maintain a relaxed stance. They were provided with visual feedback of the torque about each ankle via two analog voltmeters attached to the frame in front of them. At the start of the experiment, the torque at each rotary shaft was monitored for a period of 20 seconds and set as a baseline, along with a modifiable tolerance limit.

\section{2) Movement paradigm}

Subjects were instructed to hold down a trigger switch strapped to the right thigh, with their inner palm to initiate the trial. The torque at each foot was monitored for a period of 2 seconds by the controller, during which time subjects were required to maintain torque levels within the defined baseline operating point and tolerance limit. Once this requirement was met, the controller initiated a $10 \mathrm{~ms}$ "warn" beep from a buzzer. One second later, it illuminated an LED ("go" cue) on the frame. Subjects were instructed to react to this visual cue by executing a quick unilateral right arm raise to hit the target switch with their right fist. Subjects had mandatory 5 second rest periods between task repetitions.

In an initial practice period, subjects executed a number of trials (25-30), until a consistent repeatable movement was achieved. To this end, reaction times (delay between light onset and release of thigh switch) and movement times (delay between release of thigh switch and depression of target switch) were monitored.

\section{3) Unperturbed trials}

In approximately $25 \%$ of the trials, no perturbations were applied. These trials were used as controls to estimate changes in EMG and torques associated with the arm movement.

\section{4) Perturbed trials}

In approximately $75 \%$ of the trials, a small dorsiflexing pulse displacement ( 0.025 radians, $20 \mathrm{~ms}$ wide) was applied to the right ankle at a random delay $(800 \mathrm{~ms}-1400 \mathrm{~ms})$ relative to the piezoelectric buzzer.

Perturbed and unperturbed trials were interspersed randomly. In total, each subject executed 350-400 trials during an experiment. A mandatory 2 minute rest period was provided every 5 minutes to minimize the effects of fatigue. A total of 11 subjects participated in the study.

\section{Data Analysis and Processing}

All data were sampled at $1 \mathrm{Khz}$. Data were collected on 4 triggered, synchronized data acquisition cards (National Instruments- 4472) which had integrated anti-aliasing filters. All signals were processed in the same way; no additional post-processing was carried out on any individual channel.

\section{ANALYSIS \& RESULTS}

Data shown below are from a representative subject with the soleus used as the representative muscle for the TS complex when describing reflex and muscle activity changes.

\section{A. Unperturbed trials}

Fig. 2 shows the ensemble average of the unperturbed trials. Individual trials were aligned to the movement onset (vertical black line) using the rising edge of the thigh switch 
output as a marker of movement. This marker was found to more consistent, within a delay error of $10-15 \mathrm{~ms}$, compared to a method that used a position threshold on the kinematic data. All three muscles of the TS exhibited a characteristic APA defined by an initial inhibition followed by activation prior to movement onset. Thus, the APA onset was defined by the onset of inhibition in the soleus, indicated by the arrow in Fig. 2D. The soleus inhibition was followed by activation of the agonist TA (Fig. 2C), and finally by activation of the Deltoid (Fig. 2B) -the muscle responsible for the arm movement. In terms of the biomechanics, there was a backward shift in the COP (Fig. 2F) associated with a small dorsiflexing ankle torque change (Fig. 2E). All these changes preceded movement of the arm (Fig. 2A), which started at time zero.

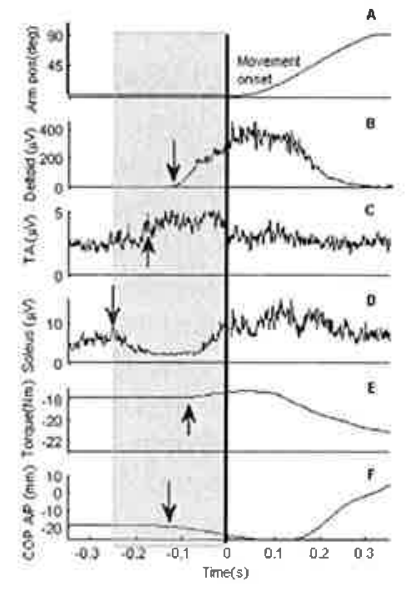

Fig. 2. Ensemble average of unperturbed trials for a typical subject. A) Arm position. B) Focal, C) TA, D) and Soleus EMG's. E) Ankle torque and F) Anterior/posterior COP. Signals were aligned on movement onset (black line). The APA region is indicated by the shaded window starting from the beginning of the soleus inhibition to movement onset. Arows indicate start of changes in each signal. Am position with $0^{\circ}$ defined as perpendicular, and $90^{\circ}$ as parallel to the floor. Dorsiflexing torques and anterior displacements of the COP are taken as positive.

\section{B. Perturbed trials}

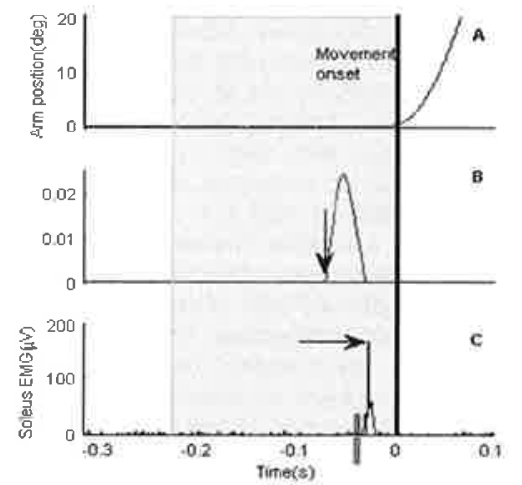

Fig. 3. Typical perturbation trial, A) Am position. B) Arkle position. C) Soleus EMG. Mean activity was recorded in a $5 \mathrm{~ms}$ window, $15 \mathrm{~ms}$ after pulse onset (blue shaded window). Movement onset was defined at time zero by the black line.
The remaining analysis focuses on the APA window, as defined. Reference data are also shown for the $100 \mathrm{~ms}$ period before and after the window.

Fig. 3 shows a representative perturbation trial. The pedal position signal was used to determine the onset of the pulse indicated by the arrow in Fig. 3B. The perturbation elicited a sharp EMG response indicated by the horizontal arrow in Fig. 3C. Note that this reflex EMG generated a reflex torque response that will be analyzed at a later date.

Perturbations were applied at different delays relative to the movement onset. The distribution of perturbation delays was chosen so that the majority occurred within the APA window. A smaller number of perturbations were applied outside the APA to act as a reference.

\section{Reflex EMG modulation}

\section{1) Invariance of Postural Control Strategy}

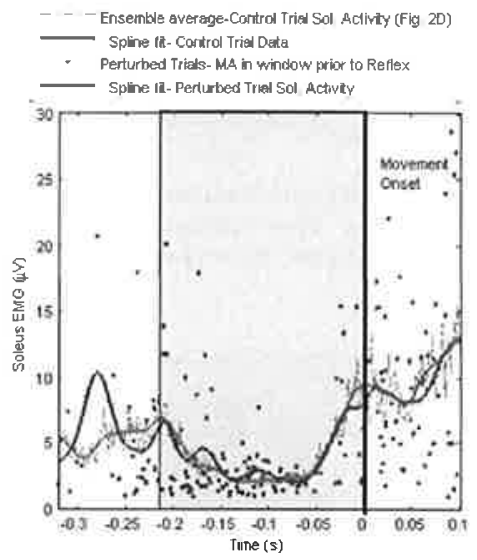

Fig. 4. Invariance of postural control strategy between unperturbed and perturbed trials. Changes in muscle activity in control (red) and perturbed (blue) trials, with overlying smoothed splines.

Subjects might have used some unintended cue to predict perturbations trials and change their postural control strategy accordingly. To test for this, we compared the EMG activity in the unperturbed and perturbed trials. Fig. 4 shows the mean EMG activity in each perturbation trial (blue windowFig. 3C) as a function of time relative to movement, as well as the underlying trend estimated by fitting a smoothing spline (blue line) to the data. Ensemble average EMG data from the unperturbed trials (Fig. 2D) are shown by the dashed red line, with a spline fitted through them as well (solid red line). As expected, the perturbed data are more variable since each point on the spline is the average of fewer trials. Nevertheless, it is evident that the two spline fits are very similar and we conclude that the same postural control strategy was used in unperturbed and perturbed trials.

2) Relative amplitude and time variation of reflex sensitivity and background muscle activity

Fig. 5 (red line) shows splines fitted to the reflex EMG 
data (Fig. 3C) as for Figure 4. The corresponding muscle activity during unperturbed trials is shown in blue. Both reflex amplitude and muscle activity were normalized to their respective ranges, and offset so that their value at APA onset was zero, to permit the relative magnitudes of their changes to be compared. The results indicate that changes in reflex and muscle activities are linked during the APA. Both parameters show a characteristic bi-phasic pattern in which there is an initial inhibition $150-200 \mathrm{~ms}$ prior to movement onset followed by a rebound $50-100 \mathrm{~ms}$ prior to onset.

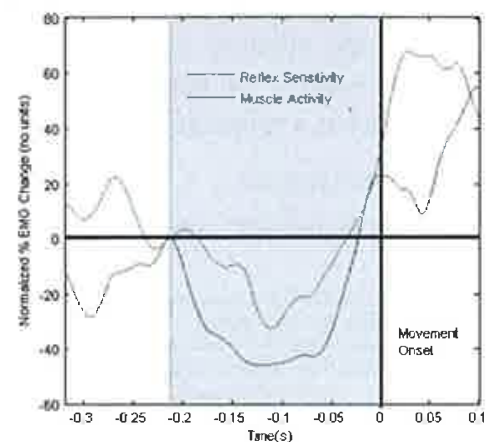

Fig. 5. Time variation of the reflex (red) and soleus muscle activity (blue) Negative values indicate down-regulation/inhibition; positive values indicate increased sensitivity/activation, relative to the start of the APA.

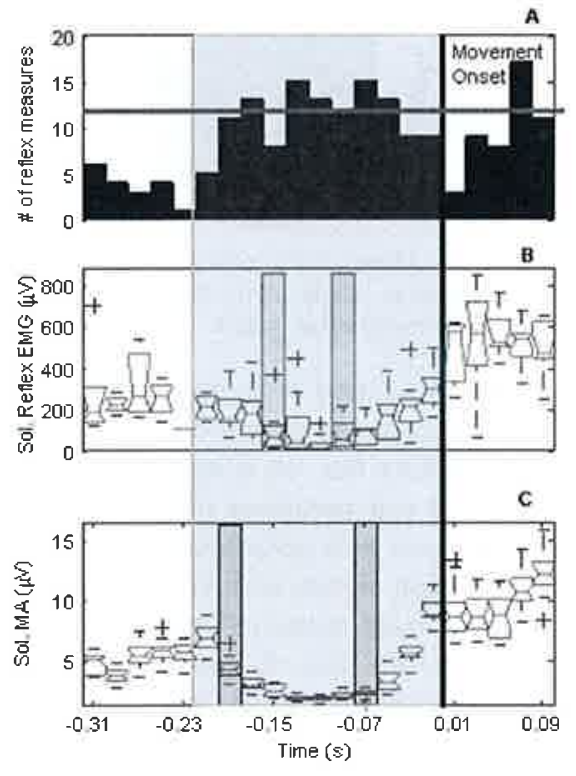

Fig. 6. Box and Whisker Plot of the Kruskal-Wallis test. A) Histogram of the number of reflex estimates in each time bin. The average in the APA is shown by the green line, B) Reflex modulation. Box boundaries are at the lower and upper quartile, encompassing the median (red). The dashed 'whisker' lines represent 1.5 times the inter-quartile range and outliers are shown by crosses. Notches on the side of each box display the variability of the median. Boxes whose notches do not overlap have different medians at the $5 \%$ significance level. C) Corresponding soleus muscle activity. Highlighted colored vertical bars show times where the onset of significant inhibition and rebound occurred for the reflex (blue bars- panel B) and muscle activity (orange bars- panel C).
However, the reflex changes seemed to be delayed with respect to those of the background muscle activity. A Kruskal-Wallis non parametric ANOVA was used to test whether these differences were statistically significant, after segmenting the raw reflex data (Fig. 6B) into $20 \mathrm{~ms}$ bins (Fig. 6A). The ensemble average of the unperturbed trials was similarly segmented (Fig. 6C). The results indicate that: 1) The oscillations in the reflex in Fig. 5 in the region prior to the APA are likely due to the low number of samples obtained at this time (Fig. 6A). Changes in the reflex and muscle activity prior to the APA were not statistically significant. 2) Reflex inhibition in the APA became significant approximately $40 \mathrm{~ms}$ after the significant decrease in muscle activity. 3) In contrast, the subsequent rebound in reflex sensitivity became significant approximately $20 \mathrm{~ms}$ before that of the muscle activity.

\section{CONCLUSION}

We found large changes in reflex excitability associated with modulations of TS muscle activity during the APA. Reflex and descending central commands that regulate the muscle activity share common efferent connections from the base of the spinal cord to the muscle. One could expect the sensitivity of the reflex to reflect the characteristic of the descending commands. However, interestingly, we found that the time course of the reflex and muscle activity changes were different with reflex inhibition lagging that of muscle activity and the subsequent rebound occurring a little earlier in the reflex. This suggests that more complex reflex control mechanisms are at work. It has been postulated that reflex sensitivities might be centrally modulated independently of associated muscle activity. Our findings support such a theory.

\section{ACKNOWLEDGMENT}

S. Vedula would like to thank the research team at REKLAB \& BVML for their invaluable input.

\section{REFERENCES}

[1] Winter, D.A., et al., Ankle muscle stiffness in the control of balance during quiet standing. J Neurophysiol, 2001. 85(6): p. 2630-3.

[2] Loram, I.D., C.N. Maganaris, and M. Lakie, Human posiural sway results from frequen, ballistic bias impulses by soleus and gastrocnemius. J Physiol, 2005. 564(Pt 1): p. 295-311.

[3] Peterka, R.J., Sensorimotor integration in human postural control. J Neurophysiol, 2002. 88(3): p. 1097-118.

[4] Morasso, P.G. and M. Schieppati, Can muscle stiffness alone stabilize upright standing? J Neurophysiol, 1999. 82(3): p. 1622-6.

[5] Gatev, P., et al., Feedforward ankle strategy of balance during quiet stance in adults. Joumal of Physiology, 1999. 514(Pt 3): p. 915-28.

[6] Fitzpatrick, R.C., J.L. Taylor, and D.I. McCloskey, Ankle stiffness of standing humans in response to imperceptible perturbation: reflex and task-dependent components. Joumal of Physiology, 1992. 454: p. 533-47.

[7] Ludvig, D., I. Cathers, and R.E. Keamey, Voluntary modulation of human stretch reflexes, Exp Brain Res, 2007. 183(2): p. 201-13.

[8] Crenna, P. and C. Frigo, A motor program for initiation of forwardoriented movements in humans. J Physiol, 1991. 437: p. 635-53. 